\title{
Resonant Elastic Scattering
}

\author{
Francois de Oliveira Santos \\ ${ }^{1}$ GANIL, CEA/DSM-CNRS/IN2P3, Boulevard Henri Becquerel, Caen Cedex 5, F-14076, France \\ e-mail: oliveira@ganil.fr
}

\begin{abstract}
Elastic scattering of nuclei at energies typically below $10 \mathrm{MeV} /$ nucleon can be used as a powerful method for studying nuclear spectroscopy. Resonances are observed in the excitation function, corresponding to unbound states in the compound nucleus. The analysis of the shape of these resonances can provide the excitation energy, the total width, the partial width, and the spin of the excited states.
\end{abstract}

\section{The Rutherford scattering}

The experimental study of nuclear collisions started when Marsden, Geiger, Rutherford and their collaborators directed alpha particles emitted by a radioactive source of radium onto different metallic foils (publication in 1909 [1]). They observed that a part of the alpha projectiles interacted "strongly" with the matter and back-scattered at large angles (beyond 90 degrees). In 1911, Rutherford published [2] an interpretation of these data. In his model, the atoms are made of a nucleus positively charged and containing most of the atomic mass, surrounding by a halo of light electrons negatively charged. The positively charged alpha particles were back-scattered by the strong Coulomb repulsion of the positively charged atomic nuclei. In 1914, Darwin [3] derived a formula for the cross section of this "Rutherford elastic scattering":

$$
\frac{d \sigma}{d \Omega}_{\text {Rutherford }}=\left(\frac{Z_{1} Z_{2} e^{2}}{E \sin \left(\frac{\theta}{2}\right)}\right)^{2}
$$

where $Z_{2}$ is the charge of the target nucleus, $E$ and $Z_{1}$ are the energy of the incident particle and its charge, scattered at center of mass angle $\theta$ (see Fig. 1 top left).

\section{First observations of anomalies in the elastic scattering}

The radioactive sources provide alpha particles with well defined energies. Different foils can be used to decelerate the particles to different lower energies. Then, it is possible to measure the excitation function, that is to say, the differential cross section as the function of the energy, and to compare it with the Rutherford scattering. According to the formula, the differential cross section should vary inversely proportional to the square of the energy. Deviations from this law were observed (Rutherford 1919 [5], Chadwick 1921 [6] ). Figure 2 shows the results obtained by Chadwick for the scattering of alpha particles onto an hydrogen target, compared to the expected results using the Rutherford scattering formula. Large deviations are observed at higher energies. 

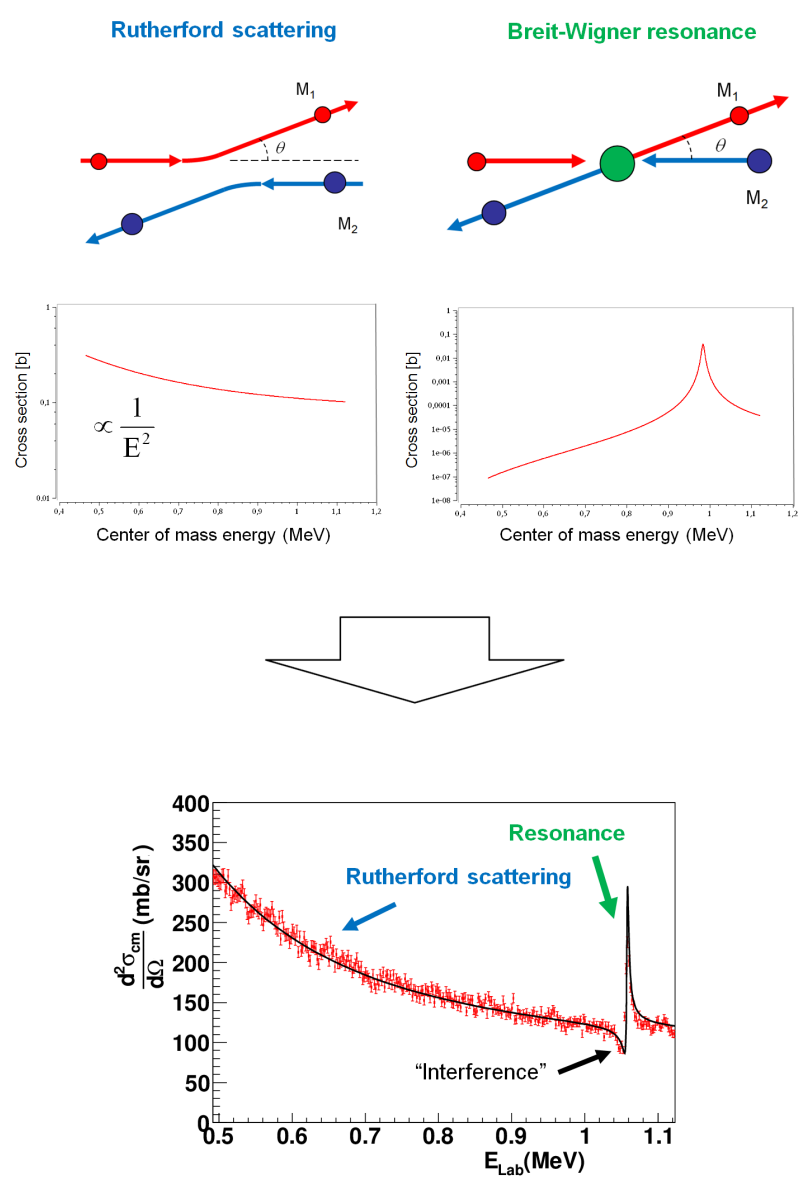

Figure 1. Top left: The Rutherford elastic scattering. The two nuclei undergo the strong Coulomb repulsion but they don't "touch" each other. The cross section can be calculated with Eq. 1. Top right: The resonant elastic scattering. The two nuclei merge and make a compound nucleus which then decays to the elastic scattering channel. The cross section can be calculated with the Breit-Wigner formula. Bottom: Excitation function of the reaction ${ }^{14} \mathrm{~N}(p, p){ }^{14} \mathrm{~N}$ measured at GANIL [4] at the angle of $180^{\circ} \mathrm{CM}$. The data follow very well the Rutherford elastic scattering formula, except at the energy close to $\mathrm{E}_{l a b} \simeq 1.06 \mathrm{MeV}$ where a resonance can be observed. It is interpreted as a resonance in the compound nucleus ${ }^{15} \mathrm{O}$. As shown at energies below the maximum of the resonance, the Rutherford and the resonant contributions interfere negatively.

\section{Resonances in the elastic scattering}

More precise measurements made later on different targets have revealed many deviations, generally localized at certain specific incident energies. For example, Fig. 1 (bottom) shows the excitation function of the elastic scattering reaction ${ }^{14} \mathrm{~N}(p, p){ }^{14} \mathrm{~N}$ measured at GANIL [4] at the angle of $180^{\circ}$ $\mathrm{CM}$. It is observed that the cross section follows very well the Rutherford elastic scattering formula, except for the energy close to $\mathrm{E}_{l a b} \simeq 1.06 \mathrm{MeV}$ where a resonance can be observed. As it is shown 


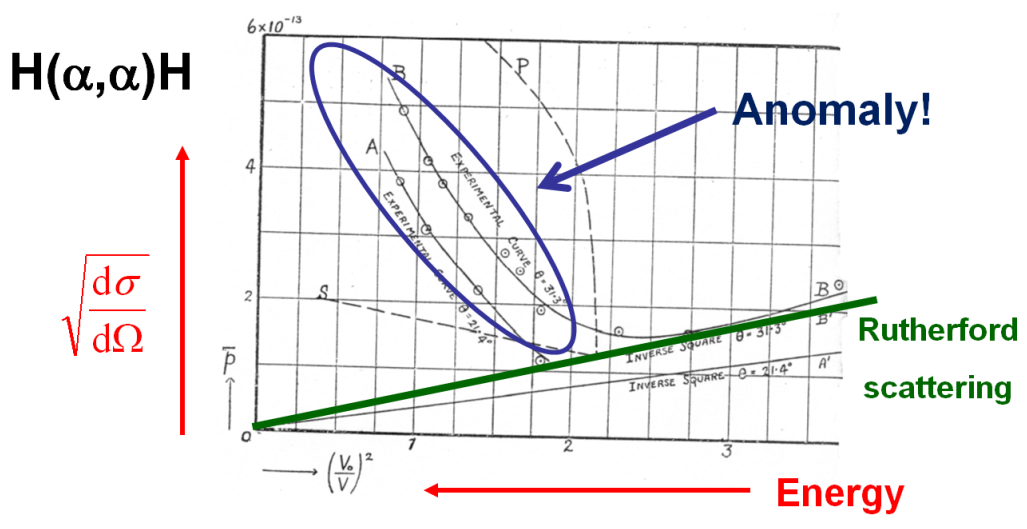

Figure 2. Square root of the differential cross section as a function of the square of the inverse of the projectile velocity, measured for the reaction $\mathrm{H}(\alpha, \alpha) \mathrm{H}$. In these axis, the Rutherford scattering formula is a straight line (green). Deviations from the Rutherford formula are observed at higher energies.

in Fig.3, this resonance matches perfectly to the energy of an excited state in the compound nucleus ${ }^{15} \mathrm{O}$.

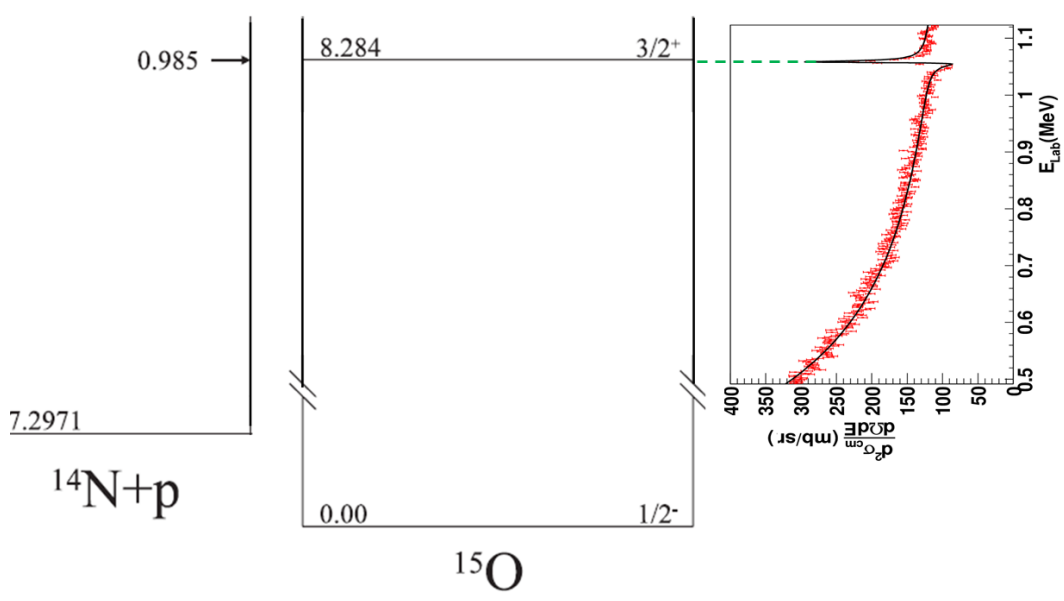

Figure 3. The energy $\mathrm{E}_{\text {lab }} \simeq 1.06 \mathrm{MeV}$ of the resonance observed in Fig. 1 , or $\mathrm{E}_{C M} \simeq 0.985 \mathrm{MeV}$ in center of mass, above the reaction threshold ${ }^{14} \mathrm{~N}+p(S=7.2971 \mathrm{MeV})$, matches perfectly to the energy $\mathrm{E}_{x}=8.284 \mathrm{MeV}$ of the $3 / 2^{+}$excited state in the compound nucleus ${ }^{15} \mathrm{O}: \mathrm{E}_{x}=S+\mathrm{E}_{C M}$. The resonance is due to the existence of this excited state.

Two different reaction mechanisms can be invoked to explain the measured data. On the one hand (Fig.1, top left), the two nuclei undergo the strong Coulomb repulsion but they don't "touch" each other. On the other hand (Fig.1, top right), the two nuclei make a compound nucleus which decays to the elastic scattering channel. This reaction mechanism is called Resonant Elastic Scattering. 


\section{R-Matrix formalism}

In reality, the two contributions, the Rutherford scattering and the resonant scattering, operate simultaneously and are indistinguishable. The final cross section is not just the sum of the two cross sections, quantum mechanics says. Amplitude of probabilities should be added, resulting sometimes into interferences between the two contributions, see Fig.1 (bottom). The R-Matrix formalism can be used to calculate, to predict or to fit the excitation function of the elastic scattering including different contributions and their interferences. The theoretical developments of this formalism would take up too much space in this article, theoretical details can be obtained in other articles, see for example Ref[7, 8]. Here, the heuristic example (without Coulomb!): the case of the s-wave scattering of a spinless neutron by a square-well potential, will be presented.

For simplicity, let's assumed the potential between the target nucleus and the projectile to be a square well with a potential depth $\mathrm{V}_{0}$ and a radius $r=a$, see Fig. 4 . The potential goes to infinity at $r=0$, and it is zero out to $r=\infty$.

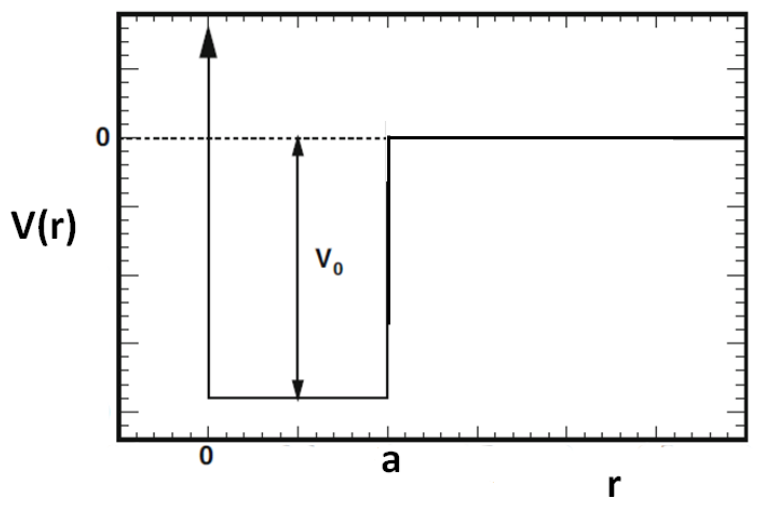

Figure 4. The potential between the target nucleus and the projectile is assumed be a square well with a potential depth $\mathrm{V}_{0}$ and a radius $r=a$.

In this simple case, two contributions are also expected: the elastic scattering from the square-well and the resonant scattering from the compound nucleus (the neutron in the mean field of the square well). The radial part of the Schrödinger equation

$$
-\frac{\hbar^{2}}{2 m} \frac{d^{2} \phi(r)}{d r^{2}}+V_{0} \phi(r)=E \phi(r)
$$

should be solved.

In the internal region $(r<a)$, the solution is

$$
\phi(r)=A \sin (K r)
$$

where $A$ is a constant and $K=\sqrt{2 m\left(E+V_{0}\right)} / \hbar$.

In the external region $(r>a)$, it is possible to show that

$$
\phi(r)=B\left[e^{-i k r}-e^{2 i \delta} e^{+i k r}\right]
$$


and the cross section of the elastic scattering

$$
\sigma(E)=4 \frac{\pi}{k^{2}} \sin ^{2}(\delta)
$$

where $B$ is a constant, $\delta$ is called the phase shift, it is a function of the energy, and $k=\sqrt{2 m(|E|)} / \hbar$.

The function $\phi(r)$ and its derivative should be continuous at $r=a$, this gives

$$
\delta=\arctan \left[\frac{k}{K} \tan (K a)\right]-k a
$$

This can be rewritten

$$
\delta=\arctan \left[k \frac{\phi(a)}{\phi(a)^{\prime}}\right]-k a
$$

The internal wave function can always be expanded with a complete set of basics states $\mathrm{X}_{\lambda}(r)$

$$
\phi(r)=\sum_{\lambda} C_{\lambda} \mathrm{X}_{\lambda}(r)
$$

where $C_{\lambda}$ are different coefficients. These coefficients can be obtained with the relation

$$
C_{\lambda}=\int_{0}^{a} \mathrm{X}_{\lambda}^{*}(r) \phi(r) d r
$$

The "resonant states" inside the square well are a good set of basics states. These "resonant states" can be obtained by solving the Schrödinger equation with the condition

$$
\frac{d \mathrm{X}_{\lambda}}{d r}(r=a)=0
$$

One obtains

$$
\mathrm{X}_{\lambda}(r)=\left(\frac{2}{a}\right)^{1 / 2} \sin \left(K_{\lambda} r\right)
$$

with

$$
K_{\lambda}=\left(\lambda+\frac{1}{2}\right) \frac{\pi}{a}
$$

At $\mathrm{r}=\mathrm{a}$, one gets

$$
\mathrm{X}_{\lambda}(r=a)=\left(\frac{2}{a}\right)^{1 / 2}
$$

Using the Schrödinger equations for $\phi(r)$ and for $\mathrm{X}_{\lambda}(r)$, one can get

$$
-\frac{\hbar^{2}}{2 m}\left\{\frac{d^{2} \phi}{d r^{2}} \mathrm{X}_{\lambda}^{*}-\phi \frac{d^{2} \mathrm{X}_{\lambda}^{*}}{d r^{2}}\right\}=\left(E-E_{\lambda}\right) \phi \mathrm{X}_{\lambda}^{*}
$$

which gives after integration

$$
-\frac{\hbar^{2}}{2 m}\left[\frac{d \phi}{d r} \mathrm{X}_{\lambda}^{*}-\phi \frac{d \mathrm{X}_{\lambda}^{*}}{d r}\right]_{0}^{a}=\left(E-E_{\lambda}\right) \int_{0}^{a} \phi \mathrm{X}_{\lambda}^{*} d r
$$

which can be used in eq. 9 , giving

$$
C_{\lambda}=\frac{\hbar^{2} / 2 m}{E_{\lambda}-E} \mathrm{X}_{\lambda}^{*}(a) \phi^{\prime}(a)
$$


Using this expression in eq.8, one gets

$$
\frac{a \phi^{\prime}(a)}{\phi(a)}=\frac{1}{\sum_{\lambda} \frac{\hbar^{2} / m a^{2}}{E_{\lambda}-E}}=\frac{1}{\mathrm{R}}
$$

where $\mathrm{R}$ is the $\mathrm{R}$-function, i.e. $\mathrm{R}=\sum_{\lambda} \frac{\gamma^{2}}{E_{\lambda}-E}$ with $\gamma^{2}=\hbar^{2} / m a^{2}$ the reduced width. Eq. 17 can be used in eq. 7 , in the approximation of a single term in $\lambda$, i.e. $\mathrm{R}=\frac{\gamma^{2}}{E_{\lambda}-E}$, giving

$$
4 \sin ^{2}(\delta)=\left|2 \sin (k a) e^{i k a}-\frac{\Gamma}{\left(E_{\lambda}-E\right)-i \frac{\Gamma}{2}}\right|^{2}
$$

with $\Gamma=2 k a \gamma^{2}$ the level width, and for the cross section (eq.5)

$$
\sigma(E)=\frac{\pi}{k^{2}}\left|2 \sin (k a) e^{i k a}-\frac{\Gamma}{\left(E_{\lambda}-E\right)-i \frac{\Gamma}{2}}\right|^{2}
$$

There are two terms in this expression. The first term corresponds to the elastic scattering onto a hard sphere (for which the potential is infinite for $r<a$ and zero for $r>a$ ). If the second term is neglected, when k goes to zero, $\sigma(E) \rightarrow 4 \pi a^{2}$, which is just the geometric cross section of the square well. The second term corresponds to the Breit-Wigner resonance. The two terms may interfere since the two amplitudes are added before putting the sum to the square.

\section{R-Matrix codes}

There are two variants of the R-matrix formalism differing mainly by their types of applications [7]. In the calculable R-matrix, the aim is to accurately solve the Schrödinger equation mostly in the continuum and predict reaction cross sections (predict resonances properties). In the phenomenological R-matrix, the goal is to parameterize scattering data (fit of data, predict cross sections from a given set of resonance properties).

Several codes exist "in the market" to calculate the scattering cross section according to the formalism of the phenomenological R-matrix, and these are often available freely. The code "AZURE" is one of the latest and best users friendly codes. Without going into details, this code is very intuitive. The particle pairs are described in a first tab (spin, separation energy etc.). The excited states of the compound nucleus are introduced as input parameters in a second tab (excitation energies, spins, widths etc.). That's about it! More details can be obtained in the reference [9].

\section{Pros and cons}

Elastic scattering reactions can be used to study the structure of the compound nucleus:

- The energies $\mathrm{E}_{x}$ of the excited states can be determine from the measured energies $\mathrm{E}_{R}$ of the resonances, since $\mathrm{E}_{x}=\mathrm{E}_{R}+S$, with $S$ the particle-emission threshold, see Fig. 3 .

- The scattering reaction is induced most often on protons, but other particles can also be used, like alpha particles.

- Due to the interferences effects, the shape of the resonances depends on the spin of the states, see Fig. 5. Then, the analysis of the shape of the resonances can be used to assign or constrain the spins of the excited states. 

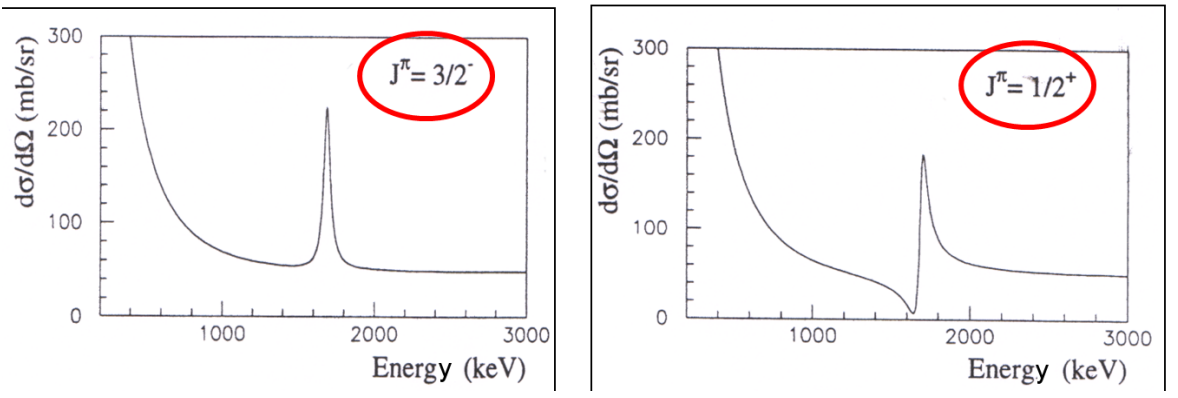

Figure 5. Elastic scattering excitation functions calculated using the same experimental conditions and exactly the same properties of the state in the compound nucleus but with different spins. The analysis of the shape of the resonance can be used to determine the spin of the states.
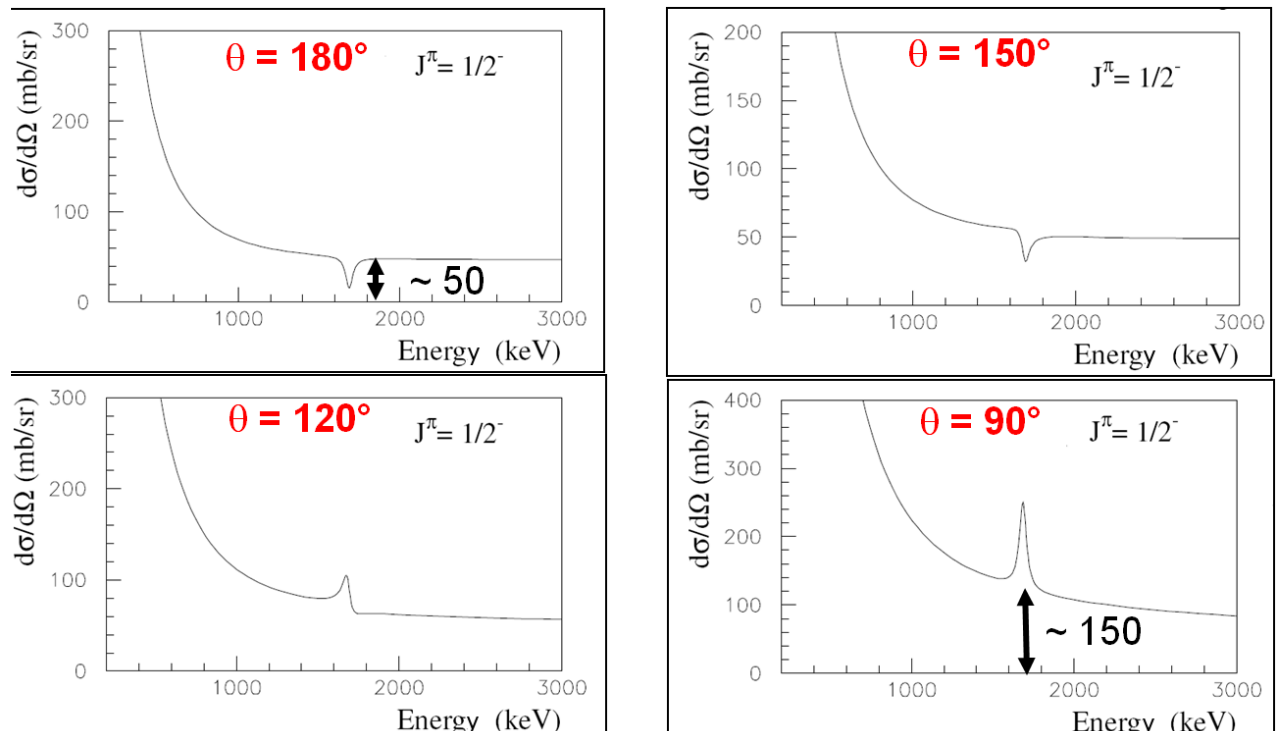

Figure 6. Elastic scattering excitation functions calculated for different angles in center of mass calculated for the reaction ${ }^{12} \mathrm{C}+$ proton (from Ref. [10]). These spectra are calculated using exactly the same properties of the resonant state in the compound nucleus. The analysis of the shape of the resonance as a function of the angle can be used to determine the spin of the state.

- The evolution of the excitation function with the angle of the scattered particles can also be used to determine the spin of the states, see Fig. 6, this is particularly useful when there are several resonances superposed.

- The width and the height of the resonances peaks can be used to measure the total and partial widths of the states.

- The differential cross sections are relatively high, typically several tens or hundreds of millibarns per steradian. Radioactive beams with relatively low intensities can be used. 
- It is possible to use a radioactive beam and to measure the elastic scattering in inverse kinematics (heavy ion onto a light target nucleus). The thick target inverse kinematics (TTIK) technique (thick enough to stop the beam inside the target), first proposed by V.Z. Goldberg [11], is very well adapted to these relatively low beams intensities. The center mass energy of the interaction point (reaction vertex) can be determined from the measured energy of the protons in the lab, after correction of the energy lost in the target. The center of mass angle is related to the lab angle by $\theta_{C M}=2 \theta_{\text {lab }}$ (this relationship is quasi-true within the special relativity). The measured cross-section has to be corrected for different effects, mainly the fact that the effective target thickness is not constant. The energy of the scattered particles in lab is related to the center of mass energy by

$$
E_{\text {lab }}(\text { scattered particle })=4 \frac{m_{p}}{m_{p}+m_{t}} E_{C M} \cos \left(\theta_{l a b}\right)
$$

with $m_{p}$ and $m_{t}$ the projectile mass and the target mass. In the case $\theta_{l a b}=0$ and a proton target, $E_{C M} \approx \frac{1}{4} E_{l a b}$ (proton). It is possible to obtain high energy resolution spectra even with a thick target (the center of mass energy resolution is about 4 times smaller than the energy resolution in the lab), see Fig. 7.

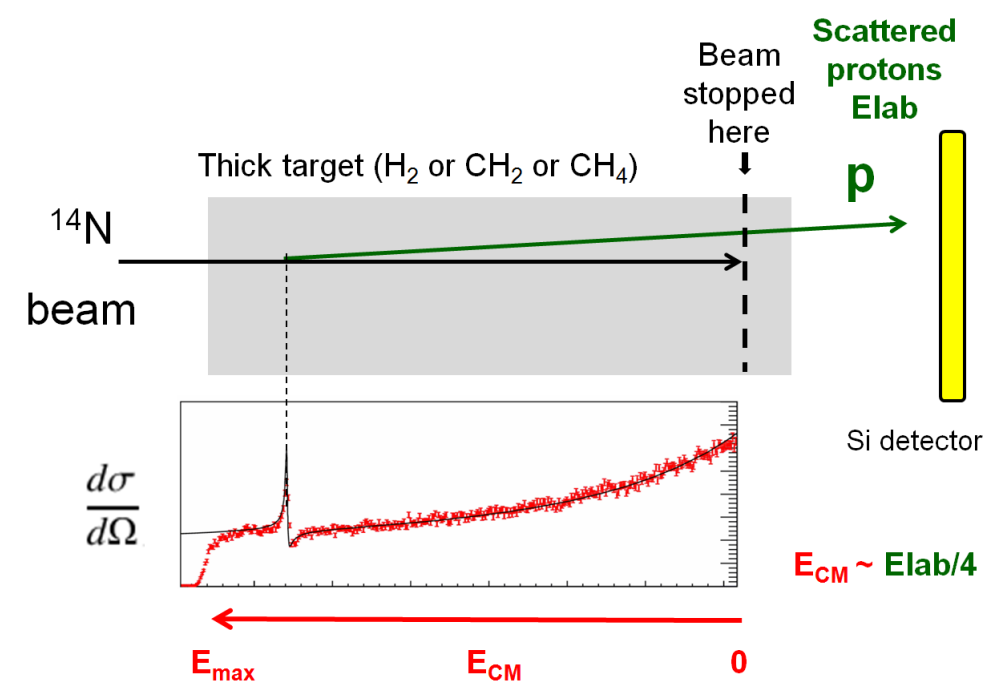

Figure 7. In the thick target technique in the case of the protons, if the energy loss of the scattered protons in the target is neglected, $E_{C M} \approx \frac{1}{4} E_{\text {lab }}$ (proton). The center of mass energy of the reaction vertex is determined from the measured energy of the protons in the lab, after correction of the small energy losses in the target.

- The energy resolution of the measured scattered protons can be estimated using the relation: $\sigma_{\text {Lab }}=\sqrt{\sigma_{\text {det }}^{2}+\sigma_{\theta}^{2}+\sigma_{\text {strag }}^{2}}$, where $\sigma_{\text {det }}$ is the energy resolution of the detector $\left(\sigma_{\text {det }}=9 \mathrm{keV}\right.$ in the ${ }^{14} \mathrm{~N}$ setting of Fig. 1), $\sigma_{\text {strag }}$ is the energy straggling in the target (estimated from simulations to be lower than $5 \mathrm{keV}$ in ${ }^{14} \mathrm{~N}$ ), and $\sigma_{\theta}$ is the energy resolution due to the aperture $d \theta$ of the detector. In inverse kinematics, it can be derived that $\sigma_{\theta}=\tan (\theta) E d \theta$. Therefore the degradation in energy resolution is minimal when $\theta=0^{\circ}$. For this reason, and for maximizing the ratio between 
the nuclear and the Coulomb contribution in the differential cross-section (see Fig. 6), the scattered protons are generally measured at very forward angles. An energy resolution of $\sigma_{L a b} \approx 10 \mathrm{keV}$ was measured in the case of ${ }^{14} \mathrm{~N}$, which lead to $\sigma_{C M} \simeq 3 \mathrm{keV}$ in the center of mass.

The resonant elastic scattering technique has some specific limitations:

- Only states above the particle emission threshold can be studied.

- The number of counts measured in the peak is proportional to the width of the resonance. The narrower it is, the more beam time is needed to observe the peak.

- When the density of states is high, it is not possible to observe isolated resonances.

- It is not possible to observe the shape of the resonance nor the spin of the state when the resonance is narrower than the experimental energy resolution. But, if a thin target is used, it is possible to determine the cross section point by point using a beam accelerated to different energies. In principle, the energy resolution could be excellent (26 eV! in ref. [12]) provided the beam has a good energy resolution.

\section{An informative experiment example}

Several cases of resonant elastic scattering experiments were presented during the 2017 Santa Tecla School [13-20]. A very informative example is given in the article of Bardayan et al [21]. Knowledge of the astrophysical rate of the ${ }^{18} \mathrm{~F}(p, \alpha){ }^{15} \mathrm{O}$ reaction is important for understanding the $\gamma$-ray emission expected from novae. The rate of this reaction is dominated at temperatures above $0.4 \mathrm{GK}$ by a resonance near $7.08 \mathrm{MeV}$ excitation energy in ${ }^{19} \mathrm{Ne}$. In this study, the authors proposed to made simultaneous measurements of the ${ }^{1} \mathrm{H}\left({ }^{18} \mathrm{~F}, p\right){ }^{18} \mathrm{~F}$ and ${ }^{1} \mathrm{H}\left({ }^{18} \mathrm{~F}, \alpha\right){ }^{15} \mathrm{O}$ excitation functions using a radioactive ${ }^{18} \mathrm{~F}$ beam at the ORNL Holifield Radioactive Ion Beam Facility. They used relatively thin target and radioactive beams accelerated to different energies. The R-Matrix analysis of the ${ }^{1} \mathrm{H}\left({ }^{18} \mathrm{~F}, p\right){ }^{18} \mathrm{~F}$ excitation function, see Fig. 8, provided many pieces of information needed to calculate the rate of the reaction. The cross section of the reaction ${ }^{1} \mathrm{H}\left({ }^{18} \mathrm{~F}, \alpha\right){ }^{15} \mathrm{O}$, combined with these pieces of information, allowed to determine the spin of the state.

\section{Acknowledgement}

This publication was supported by OP RDE, MEYS Czech Republic under the project EF16013/0001679, by LEA NuAG, by LIA COSMA and by LIA COLL-AGAIN.

\section{References}

[1] H. Geiger, E. Marsden, Proceedings of the Royal Society of London Series A 82, 495 (1909)

[2] E. Rutherford, Phil. Mag. 6, 21 (1911)

[3] C. Darwin, Phil. Mag. XXVII, 499 (1914)

[4] I. Stefan, Ph.D. thesis (Université de Caen, France, 2006)

[5] Rutherford, Phil. Mag. XXXVII, 537 (1919)

[6] J. Chadwick, Phil.Mag. 42, N252, 923 (1921)

[7] P. Descouvemont, D. Baye, Reports on progress in physics 73, 036301 (2010)

[8] C.A. Bertulani, Nuclei in the Cosmos (World Scientific, 2014)

[9] R. Azuma, E. Uberseder, E. Simpson, C. Brune, H. Costantini, R. De Boer, J. Görres, M. Heil, P. LeBlanc, C. Ugalde et al., Physical Review C 81, 045805 (2010) 


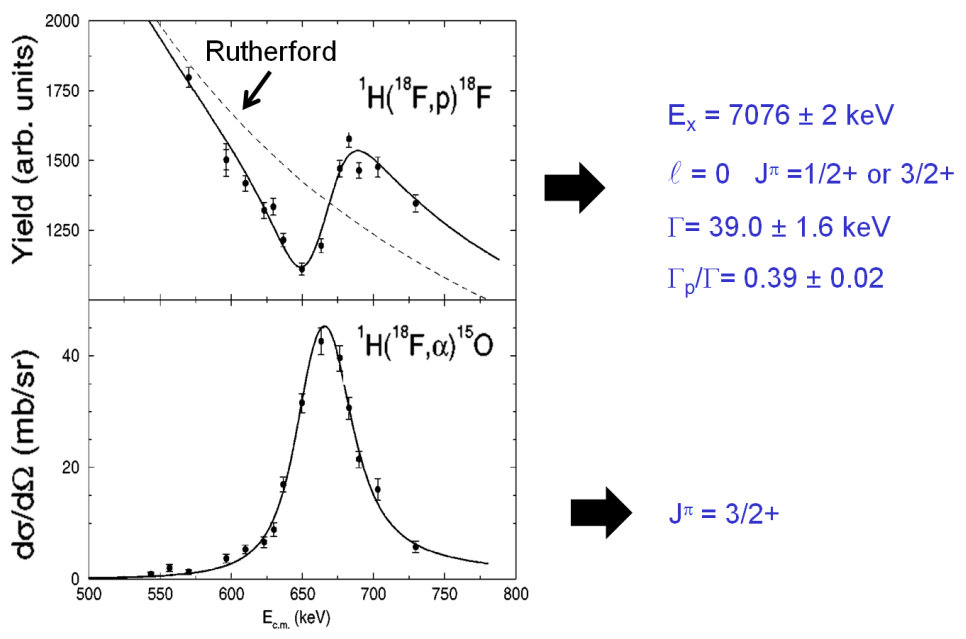

Figure 8. The reactions ${ }^{1} \mathrm{H}\left({ }^{18} \mathrm{~F}, p\right){ }^{18} \mathrm{~F}$ and ${ }^{1} \mathrm{H}\left({ }^{18} \mathrm{~F}, \alpha\right){ }^{15} \mathrm{O}$ were measured in the same experiment simultaneously [21]. The R-matrix formalism was used to fit the two spectra. Important spectroscopic information were extracted.

[10] Lynda Achouri (Ph. D Thesis. Universite de Caen, 2001)

[11] V. Gol’Dberg, A. Pakhomov, Physics of Atomic Nuclei 56, 1167 (1993)

[12] S. Wüstenbecker, H. Becker, H. Ebbing, W. Schulte, M. Berheide, M. Buschmann, C. Rolfs, G. Mitchell, J. Schweitzer, Zeitschrift für Physik A Hadrons and nuclei 344, 205 (1992)

[13] D. Torresi, L. Cosentino, P. Descouveont, A. Di Pietro, C. Ducoin, P. Figuera, M. Fisichella, M. Lattuada, C. Maiolino, A. Musumarra et al., 8Li+ $\alpha$ resonant elastic scattering: a tool to study cluster states in 12B, in Journal of Physics: Conference Series (IOP Publishing, 2014), Vol. 569, p. 012024

[14] H. Yamaguchi, D. Kahl, Y. Wakabayashi, S. Kubono, T. Hashimoto, S. Hayakawa, T. Kawabata, N. Iwasa, T. Teranishi, Y. Kwon et al., Physical Review C 87, 034303 (2013)

[15] F. de Oliveira Santos, P. Himpe, M. Lewitowicz, I. Stefan, N. Smirnova, N. Achouri, J. Angélique, C. Angulo, L. Axelsson, D. Baiborodin et al., The European Physical Journal A-Hadrons and Nuclei 24, 237 (2005)

[16] M. Freer, E. Casarejos, L. Achouri, C. Angulo, N. Ashwood, N. Curtis, P. Demaret, C. Harlin, B. Laurent, M. Milin et al., Physical review letters 96, 042501 (2006)

[17] L. Axelsson, M.J.G. Borge, S. Fayans, V.Z. Goldberg, S. Grévy, D. Guillemaud-Mueller, B. Jonson, K.M. Källman, T. Lönnroth, M. Lewitowicz et al., Phys. Rev. C 54, R1511 (1996)

[18] M. Assie, F. de Oliveira Santos, T. Davinson, F. De Grancey, L. Achouri, J. Alcántara-Núñez, T. Al Kalanee, J.C. Angélique, C. Borcea, R. Borcea et al., Physics Letters B 712, 198 (2012)

[19] I. Stefan, F. de Oliveira Santos, O. Sorlin, T. Davinson, M. Lewitowicz, G. Dumitru, J. Angélique, M. Angélique, E. Berthoumieux, C. Borcea et al., Physical Review C 90, 014307 (2014)

[20] F. De Grancey, A. Mercenne, F. de Oliveira Santos, T. Davinson, O. Sorlin, J. Angélique, M. Assié, E. Berthoumieux, R. Borcea, A. Buta et al., Physics Letters B 758, 26 (2016)

[21] D. Bardayan et al., Phys. Rev. C 63, 065802 (2001) 